\title{
Reduced partition function ratios for isotopes of hydrated alkali and alkaline earth ions calculated by a simple electrostatic model
}

\author{
Maciej Czarnacki, Stanislaw Halas \\ Mass Spectrometry Laboratory, Institute of Pysics, \\ Marie Curie-Sklodowska University, Lublin
}

\begin{abstract}
We describe a simple electrostatic model of hydrated ions $\left[\mathrm{M}\left(\mathrm{H}_{2} \mathrm{O}\right)_{n}\right]^{+}(\mathrm{n}=3-18, \mathrm{M}=\mathrm{Li}, \mathrm{Mg}, \mathrm{Ca}$, $\mathrm{K})$ which enables to calculate ion vibration frequency of the ground state. In this model the considered ion with a reduced mass vibrates in quasispherical well formed by the ion-dipole attractive potential and repulsive valence potential, these simplifications allowed to solve one dimensional Schrödinger equation, whilst the calculated ground state was considered as one of triply degenerated state of the three dimensional motion of the ion vs. hydration shell. The reduced partition function ratios were calculated from the vibration frequencies using Urey's (1947) harmonic approximation formula.

The results obtained in this way are in good agreement with those obtained by much more laborious ab initio molecular orbital methods, like SCF Hartree-Fock, DFT, MP2, etc. Moreover, we were able to extend calculations to hydrated $\mathrm{Li}$ and $\mathrm{K}$ ions surrounded with two shells of water molecules. These results are the first estimations of the upper limit of isotope fractionation in water solutions, which are $99.3 \%$ for $\mathrm{Li}$ and only $2.5 \%$ for $\mathrm{K}$ isotopes.
\end{abstract}

Key words: hydration, ion radii, isotope effects, $\mathrm{Li}, \mathrm{Mg}$, $\mathrm{Ca}, \mathrm{K}$, vibration frequencies, $\beta$ factors

\section{Introduction}

For more than a decade precise analytical methods (TIMS and ICPMS) for the study of natural variations of lithium isotopes have been developed (Tomascak 2004 and references therein). These studies recognized that natural variation of ${ }^{7} \mathrm{Li} /{ }^{6} \mathrm{Li}$ isotope ratios exceeds $60 \%$ o. In contrast the variation of the ${ }^{41} \mathrm{~K} /{ }^{39} \mathrm{~K}$ ratio in terrestrial materials is at the $0.5 \%$ level (Humayun and Clayton 1995), which is surprising for a mass dependent isotope effect at the relative mass differences of

Addresses: M. Czarnacki, S. Halas: Marie Curie-Sklodowska University, 20-031 Lublin, Poland e-mails: maciej.czarnacki@gmail.com, halas@tytan.umcs.lublin.pl

Received: April 4, 2011; accepted: June 8, 2011 
$(7-6) / 6=0.16$ and $(41-39) / 39=0.05$, respectively. The answer to this puzzle relates to the hydration of these elements.

Lithium has the smallest cation radius, but the largest radius of the hydration sphere. For this reason the electric interaction of $\mathrm{Li}^{+}$with the inner shell of dipoles of water molecules is particularly strong and it leads to such a great isotope fractionation. In this paper we will compare the hydration effect in the case of $\mathrm{Li}, \mathrm{K}, \mathrm{Mg}$ and $\mathrm{Ca}$ cations. We have extended calculation of reduced partition function ratios to these of $\mathrm{Mg}$ and Ca cations in order to test our model by comparison with the results obtained by more sophisticated ab initio methods (Rustad et al. 2010).

The first calculation of equilibrium constant for isotope exchange reaction between lithium hydrides ( $\mathrm{LiH}$ and $\mathrm{LiD}$ ) and lithium vapour for a broad temperature range was reported by Urey (1947): at $298.1 \mathrm{~K}$ the equilibrium constants attain 1.025 and 1.029, respectively. Lithium isotope exchange reaction between solid $\mathrm{Li}$ and dissolved $\mathrm{LiCl}$ was experimentally determined by Singer and Rock (1972). However, their equilibrium constant $(\mathrm{K}=1.046 \pm 0.013)$ was determined electrochemically with low precision and for one temperature (298.6K) only. They also tried to calculate this equilibrium constant from statistical thermodynamics, but their value was evidently too large (1.29-1.33). Theoretical estimation of lithium isotopic reduced partition function ratio for lithium ions in aqueous solution were made by Yamaji et al. (2001), who report their ab initio results for only one temperature $(298 \mathrm{~K})$ : their maximum value of lithium isotope fractionation factor between hydrated ion and " $\mathrm{Li}^{+}$exchanger" is 1.07 .

\section{Model}

We will consider a metal ion electrically interacting with surrounding electric dipoles of water molecules. Such electrostatic approach is fully justified because the ion motion is much slower in comparison to the electronic motion in both water molecule and ion. Geometry of the electrostatic model used in calculations is shown in Fig. 1. Numerical values for the water molecule were taken from Malenkov (1962).

Let $p$ denote dipole moment of the water molecule, then the electric potential at distance $r$ from its center (for $r>>$ distance between negative, $-q$, and positive, $+q$, charge making up this dipole) in polar coordinates is

$$
V(r, \varphi)=\frac{p}{4 \pi \varepsilon_{0}} \frac{\cos \varphi}{r^{2}}
$$

where $\mathrm{f}$ is the angle between vector $(-q,+q)$ and the vector $\mathrm{r}$, and $\varepsilon_{\mathrm{o}}$ is the electric constant.

Formula (1) may be rewritten in Cartesian coordinates, taking into account that for any plane $(\mathrm{x}, \mathrm{y})$ passing along the vector $(-q,+q)$ we have $r^{2}=x^{2}+y^{2}$, and $\cos \varphi x / r$ 


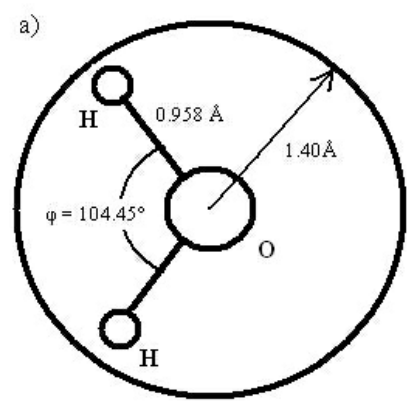

water molecule model

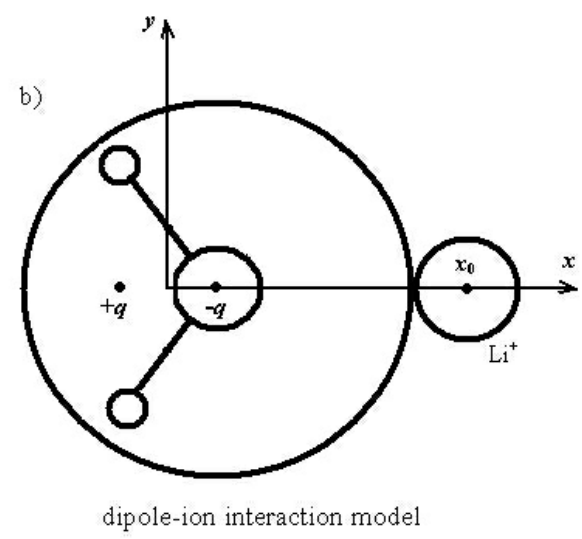

Fig. 1

Geometry of the electrostatic model used in calculations

Hence

$$
V(x, y)=\frac{p}{4 \pi \varepsilon_{0}} \frac{x}{\left(y^{2}+x^{2}\right)^{\frac{3}{2}}}
$$

In our model $\mathrm{x}$ will be fixed to an $\mathrm{x}_{0}$ value at which the ion sphere touches that of oxygen atom in $\mathrm{H}_{2} \mathrm{O}$ molecule, thereby the stationary (time independent) states of the ion motion can be found from the Schödinger equation

$$
\hat{H}(y) \psi(y)=E \psi(y)
$$

Where $\hat{H}(y)$ is the Hamiltonian and $\mathrm{E}$ is the energy of the state represented by the wave function $\psi(\mathrm{y})$

In order to account for the total energy from interaction of the ion with $\mathrm{k}$ dipoles in the first shell (at the distance $x_{0}$ ) and 1 dipoles in the second shell (at a larger distance $x_{1}$ ) the potential energy of the Hamiltonian was extended as follows:

$$
\hat{H}(y)=\frac{h^{2}}{2 \mu} \nabla^{2}-\frac{e k p}{4 \pi \varepsilon_{0}} \frac{x_{0}}{\left(y^{2}+x_{0}^{2}\right)^{3}}-\frac{e l p}{4 \pi \varepsilon_{0}} \frac{x_{1}}{\left(y^{2}+x_{1}^{2}\right)^{3}}
$$

where $\mu$ is the reduced mass of the ion surrounded by $k$ water molecules in the first shell of the hydration sphere and it was expressed by the mass of ion and mass of oxygen of the adjacent $\mathrm{k}$ water molecules:

$$
\mu=\frac{m_{M} \cdot k m_{O}}{m_{M}+k m_{O}}
$$


The $\mathrm{x}_{0}$ values given in Table 1 are defined as the distances between the considered cation center and dipole center of water molecules from the first shell, note that the last center differs from the water molecule center treated as a ball of radius given in Table 1 . In similar manner are defined distances $\mathrm{x}_{1}$, though not shown in Fig. 1. In calculations of the distances $x_{0}$ and $x_{1}$, we anticipated that water dipoles are uniformly distributed in the space around a considered metal ion due to their mutual repulsion. In calculations of the reduced mass (Table 2), only the first shell of dipoles was taken into account, because the second one has a weak interaction and is more elastically bounded to the ion.

The Schrödinger equation (3) with the Hamiltonian (4) was solved in order to calculate the ground state, i.e. zero point energy (ZPE) level. The lowest state may be considered as that of quantum oscillator state, because the resulting potential energy of the considered metal ion surrounded by one or two shells of water dipoles has a parabolic character, see plots in Fig. 2.

For the ZPE calculation we used the computer program MARRS (Salejda et al. 2002), the algorithm of which was based on the finite elements method with 5 points approximation of the second derivative. Calculations of the wave functions, $\psi$, and the energy states were made on grids composed of 64000 elements. The parameters used in this model are listed in Table 1, the reduced masses are listed in Table 2, whereas in Table 3 are given the calculated vibrational frequencies (in $\mathrm{cm}^{-1}$ units) of the central ion of each considered cluster (for heavy and light isotope, respectively).

Table 1

Values of parameters used in ion-water dipole interaction model calculations

\begin{tabular}{cccccc}
\hline Parameter & $\mathrm{H}_{2} \mathrm{O}$ & $\mathrm{Li}^{+}$ & $\mathrm{Mg}^{2+}$ & $\mathrm{Ca}^{2+}$ & $\mathrm{K}^{+}$ \\
\hline Radius $[\AA]$ & 1.4 & $0.38^{3}$ & $0.66^{2}$ & $0.99^{2}$ & $1.33^{2}$ \\
Charge [e] & 0 & +1 & +2 & +2 & +1 \\
$x_{0}[\AA]$ & - & 2.08 & 2.35 & 2.68 & 3.02 \\
$x_{1}[\AA]$ & - & 3.41 & 3.43 & 3.47 & 3.59 \\
Dipole moment [D] & $1.8546^{1}$ & - & - & - & - \\
\hline${ }^{1}$ Clough et al. 1973, ${ }^{2}$ Weast $1980,{ }^{3}$ Halas and Mackiewicz 2003 &
\end{tabular}

Table 2

Reduced mass of cations used in our calculations expressed in atomic mass units

\begin{tabular}{c|cccc|cc|cc}
\hline$k$ & ${ }^{6} \mathrm{Li}^{+}$ & ${ }^{7} \mathrm{Li}^{+}$ & ${ }^{24} \mathrm{Mg}^{2+}$ & ${ }^{26} \mathrm{Mg}^{2+}$ & ${ }^{40} \mathrm{Ca}^{2+}$ & ${ }^{44} \mathrm{Ca}^{2+}$ & ${ }^{39} \mathrm{~K}^{+}$ & ${ }^{41} \mathrm{~K}^{+}$ \\
\hline 3 & 5.33 & 6.11 & - & - & - & - & - & - \\
4 & 5.48 & 6.31 & - & - & - & - & - & - \\
5 & 5.33 & 6.11 & - & - & - & - & - & - \\
6 & 5.64 & 6.52 & 19.20 & 20.46 & 28.23 & 30.17 & 27.73 & 28.73 \\
\hline
\end{tabular}




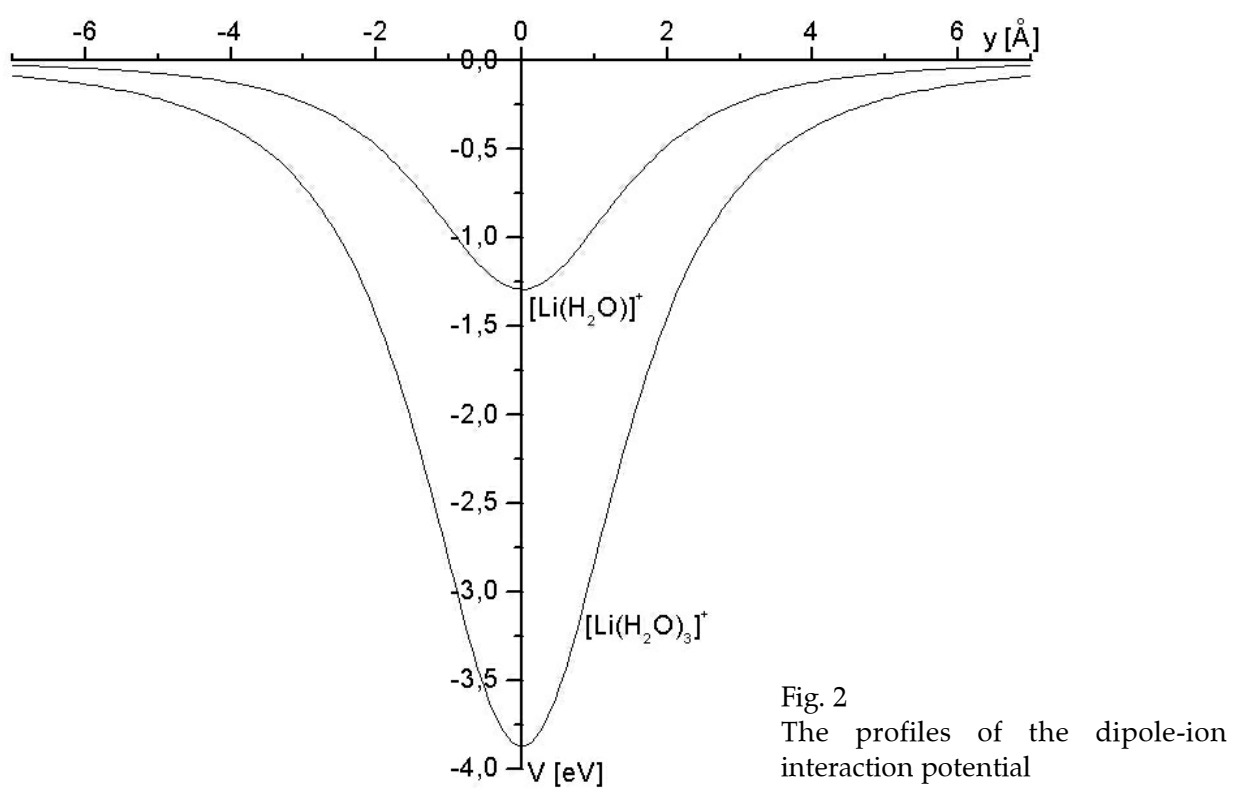

\section{Calculation of $\beta$ factors for hydrated ions}

Having calculated ZPE levels of the vibration motion of the ion, we can calculate the $\beta$ factors, i.e. reduced partition function ratios, which are fundamental in calculation of the isotope effects at thermodynamic equilibrium. The $\beta$ factor is defined as follows:

$$
\beta=\frac{Q_{t r}^{H} Q_{r o t}^{H} Q_{v i b}^{H}}{Q_{t r}^{L} Q_{r o t}^{L} Q_{v i b}^{L}}
$$

where the upper subscripts, $\mathrm{H}$ and $\mathrm{L}$, refer to the appropriate partition function, $\mathrm{Q}$, of the isotopically substituted hydrated ion by heavy and light isotope, respectively, whereas the lower subscripts denote translational, rotational and vibration degree of freedoms. In the harmonic approximation the formula (6) can be converted to the following (Urey 1947, O'Neil 1986, Chacko et al 2001):

$$
\beta=\Pi_{i} \frac{u_{i}^{H}}{u_{i}^{L}} \frac{\exp \left(-\frac{u_{i}^{H}}{2}\right)\left(1-\exp \left(-u_{i}^{L}\right)\right)}{\left(1-\exp \left(-u_{i}^{H}\right)\right) \exp \left(-\frac{u_{i}^{L}}{2}\right)}
$$


where $u j=2 \cdot E_{j} /\left(k_{B} T\right), E_{j}$ is the ZPE value of the $j$-th vibration mode, $k_{B}$ is the Boltzmann constant and $T$ is absolute temperature.

The product in equation (7) is extended over all vibration modes. It should be noted that this product is reduced to the three vibration states of the hydrated ion only, because the remaining vibrations in the considered system are insensitive to isotope substitution of the central ion. In the case of highly symmetric system, which is the case for high hydration degree, we may safely assume that all the three vibrational modes (the motion proceeds in the 3dimensional space) are identical. This assumption is equivalent to triple degeneration of the energy states of the full 3-dimensional Schrödinger equation, which should be solved instead of equation (3).

\section{Results}

The $\beta$ factors for the following isotopically substituted systems of $\left[\mathrm{Li}\left(\mathrm{H}_{2} \mathrm{O}\right)_{n}\right]^{+}$, $\left[\mathrm{Mg}\left(\mathrm{H}_{2} \mathrm{O}\right)_{n}\right]^{2+}$, $\left[\mathrm{Ca}\left(\mathrm{H}_{2} \mathrm{O}\right)_{n}\right]^{2+}$ and $\left[\mathrm{K}\left(\mathrm{H}_{2} \mathrm{O}\right)_{n}\right]^{+}$were calculated by the method presented above and compared with those existing literature data (Yamaji et al. 2001, Rustad et al. 2010).

In Table $3 \beta$ factors calculated for temperature $273.15 \mathrm{~K}$ are shown for $\left[\mathrm{M}\left(\mathrm{H}_{2} \mathrm{O}\right)_{n}\right]^{+}$clusters with different number of water molecules, $n=k+l$, distributed in two shells. In frame-work of the electrostatic model we could straightforward consider the influence of water dipoles from the second shell of the hydrated ion. It should be noted that for hydrated ion of potassium, $\left[\mathrm{K}\left(\mathrm{H}_{2} \mathrm{O}\right)_{n}\right]^{+}$, no evaluations of $\beta$ factors were published by far.

The comparison of calculated reduced partition function ratios with literature data is shown in Table 4. It is seen that our values for lithium isotopes are close to the first set of results calculated by Yamaji et al. (2001) or fall between their two sets. The same refers to $\beta$ factors for magnesium and calcium isotopes, which are compared with results of Rustad et al. (2010). The calculated $1000 \cdot \ln (\beta)$ values as a function of temperature are listed in Table 5 and plotted in Fig. 3.

\section{Discussion}

The calculations made in this paper refer to highly symmetric systems of the hydrated ions of alkali and alkaline earth metals. These systems within electrostatic approximation attain nearly spherical symmetry due to dominant electrostatic interactions between $\mathrm{H}_{2} \mathrm{O}$ dipoles and considered spherical ions having closed electron shells.

Below we will discuss the influence of the parameters used in our model (Tables 1 and 2) on the final result. We will allow varying the most important parameters (ion radius, reduced mass, and dipole moment of water molecule) in calculation of $\beta$ factor for lithium, which is the most sensitive cation to hydration isotope effects. These calculations have been made for $\left[\mathrm{Li}\left(\mathrm{H}_{2} \mathrm{O}\right)_{n}\right]^{+}$, where $\mathrm{n}=6$ 
Table 3

Values of the reduced partition function ratio, $\beta$, at $273.15 \mathrm{~K}$ for $\left[\mathrm{M}\left(\mathrm{H}_{2} \mathrm{O}\right)_{n}\right]^{+}$clusters. $\mathrm{k}$ is the number of water molecules in the first hydration shell and 1 is the number of water molecules in the second shell, $\mathrm{v}^{\mathrm{H}}$ and $\mathrm{v}^{\mathrm{L}}$ are triply degenerated vibrational frequencies of the central ion (of heavy and light isotope, respectively) in each considered cluster

\begin{tabular}{cccccc}
\hline Cluster type & $k$ & $l$ & $\begin{array}{c}v^{H} \\
{\left[\mathrm{~cm}^{-1}\right]}\end{array}$ & $\begin{array}{c}v^{L} \\
{\left[\mathrm{~cm}^{-1}\right]}\end{array}$ & $\beta$ \\
\hline$\left[\mathrm{Li}\left(\mathrm{H}_{2} \mathrm{O}\right)_{\mathrm{n}}\right]^{+}$ & 3 & 0 & 347.64 & 371.91 & 1.059 \\
& 4 & 0 & 395.29 & 423.77 & 1.078 \\
& 5 & 0 & 403.28 & 431.43 & 1.079 \\
& 6 & 0 & 397.46 & 427.03 & 1.082 \\
& 6 & 8 & 437.32 & 469.80 & 1.098 \\
& 6 & 12 & 454.81 & 488.56 & 1.104 \\
\hline$\left[\mathrm{Mg}\left(\mathrm{H}_{2} \mathrm{O}\right)_{\mathrm{n}}\right]^{2+}$ & 6 & 0 & 300.16 & 309.69 & 1.020 \\
& 6 & 8 & 333.15 & 343.67 & 1.024 \\
{$\left[\mathrm{Ca}\left(\mathrm{H}_{2} \mathrm{O}\right)_{\mathrm{n}}\right]^{2+}$} & 6 & 12 & 346.34 & 357.28 & 1.026 \\
& 6 & 0 & 190.30 & 196.61 & 1.008 \\
{$\left[\mathrm{~K}\left(\mathrm{H}_{2} \mathrm{O}\right)_{\mathrm{n}}\right]^{+}$} & 6 & 8 & 221.89 & 229.20 & 1.011 \\
& 6 & 12 & 234.85 & 242.56 & 1.013 \\
& 6 & 0 & 108.27 & 110.17 & 1.0014 \\
& 6 & 8 & 133.88 & 136.22 & 1.0022 \\
& 6 & 12 & 143.23 & 145.74 & 1.0025 \\
\hline
\end{tabular}

+12 . For $\mathrm{r}_{\mathrm{Li}}=0.68 \AA$, at the remaining parameters conserved, one obtains $\beta=$ 1.091, which is considerably lower than result 1.104 presented in Table 3, but still it looks sensible. However we have intentionally used the revised value of $0.382 \AA$ from Halas and Mackiewicz (2003) as the most reliable, because it is consistent with other ionic radii for two electron systems: $\mathrm{H}^{-}, \mathrm{He}, \mathrm{Li}^{+}, \mathrm{Be}^{2+}, \mathrm{B}^{3+} \ldots$ Another independent estimation of $\mathrm{Li}^{+}$radius follows from consideration of the length of nearly pure ionic bounding in the $\mathrm{LiCl}$ molecule, which is 2.01-2.03 $\AA$ (Pauling 1960, Boldyrev and Simson 1993). Calculating the length of ionic bounding in $\mathrm{LiCl}$ molecule with $\mathrm{Li}^{+}$radius taken from CRC Handbook one obtains $2.49 \AA$ whereas with that of Halas and Mackiewicz (2003) one obtains a more

Table 4

Comparison of calculated reduced partition function ratios with literature data. $\mathrm{N}$ is total number of water molecules in the considered cluster

\begin{tabular}{cccc}
\hline $\mathrm{N}$ & This work & \multicolumn{2}{c}{ Literature } \\
\hline \multicolumn{4}{c}{$\mathrm{Li}^{+} \mathrm{T}=298^{\circ} \mathrm{K}$} \\
3 & 1.050 & $1.059^{1}$ & $1.067^{1}$ \\
4 & 1.066 & $1.067^{1}$ & $1.070^{1}$ \\
5 & 1.066 & $1.074^{1}$ & $1.056^{1}$ \\
6 & 1.069 & $1.081^{1}$ & $1.041^{1}$ \\
\hline \multicolumn{4}{c}{$\mathrm{Mg}^{2+} \mathrm{T}=273.15^{\circ} \mathrm{K}$} \\
14 & 1.024 & - & - \\
18 & 1.026 & $1.027^{2}$ & $1.028^{2}$ \\
\hline \multicolumn{4}{c}{$\mathrm{Ca}^{2+} \mathrm{T}=273.15^{\circ} \mathrm{K}$} \\
14 & 1.011 & - & - \\
18 & 1.013 & $1.016^{2}$ & $1.017^{2}$ \\
21 & - & $1.013^{2}$ & $1.014^{2}$ \\
\hline${ }^{1}$ Yamaji et al. $2001,{ }^{2} \mathrm{Rustad}$ et al. 2010.
\end{tabular}




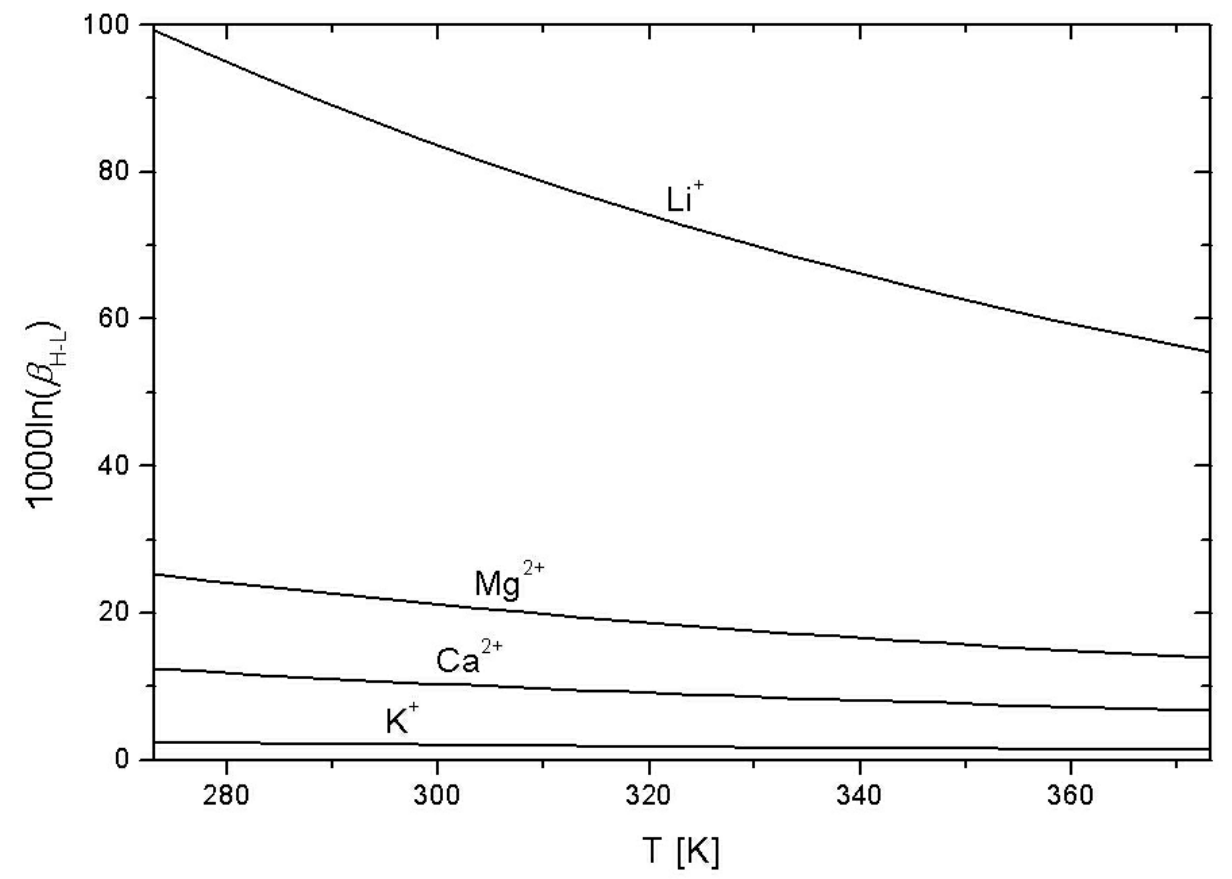

Fig. 3

Permil fractionation between hydrated ion and respective metal vapor as a function of temperature

reliable value of $2.19 \AA$ Similar consideration for ionic bounding in the $\mathrm{LiF}$ molecule yields $1.71 \AA$ with $\mathrm{Li}^{+}$radius taken from Halas and Mackiewicz (2003) and $2.01 \AA$ with $\mathrm{Li}+$ radius taken from CRC Handbook, whilst Boldyrev and Simson (1993) report $1.59 \AA$ (ab initio calculation) and $1.56 \AA$ (experimental result). These two examples of ionic bounding support the revised value for $\mathrm{Li}^{+}$ radius.

The next parameter, $\mu$, was calculated assuming that only oxygen in $\mathrm{H}_{2} \mathrm{O}$ is moving during ion vibration, which seems sensible. However with mass of the whole water molecule, we obtain for $\mu$ values 5.68 and 6.57 a.u., respectively, and identical $\beta=1.104$ as in Table 3. Finally we have considered the uncertainty of dipole moment of $\mathrm{H}_{2} \mathrm{O}$ molecule. Assuming $\mathrm{p}=3 \mathrm{D}$ for liquid water instead 1.85 D one obtains obviously too large $\beta=1.165$; such high value for $\mathrm{p}$ was calculated by Silvestrelli and Parrinello (1999), however it has not been determined experimentally.

The advantages and drawbacks of our approach in comparison with ab initio methods may be summarized as follows. The new method is highly effective for fast evaluation of the reduced partition function ratios for hydrated ions. The results obtained by this method are in good agreement with those obtained by 
Table 5

Calculated $1000 \cdot \ln (\beta)$ values as a function of temperature for all the considered hydrated ions

\begin{tabular}{|c|c|c|c|c|}
\hline \multicolumn{5}{|c|}{$1000 \cdot \ln (\beta), \quad \mathrm{n}=18$} \\
\hline $\mathrm{T}\left[{ }^{\circ} \mathrm{C}\right]$ & {$\left[\mathrm{Li}\left(\mathrm{H}_{2} \mathrm{O}\right)_{\mathrm{n}}\right]^{+}$} & {$\left[\mathrm{Mg}\left(\mathrm{H}_{2} \mathrm{O}\right)_{\mathrm{n}}\right]^{2+}$} & {$\left[\mathrm{Ca}\left(\mathrm{H}_{2} \mathrm{O}\right)_{\mathrm{n}}\right]^{2+}$} & {$\left[\mathrm{K}\left(\mathrm{H}_{2} \mathrm{O}\right)_{\mathrm{n}}\right]^{+}$} \\
\hline 0 & 99.3 & 25.3 & 12.4 & 2.48 \\
\hline 10 & 93.0 & 23.6 & 11.6 & 2.31 \\
\hline 20 & 87.2 & 22.1 & 10.8 & 2.16 \\
\hline 25 & 84.5 & 21.4 & 10.5 & 2.08 \\
\hline 30 & 81.9 & 20.7 & 10.1 & 2.02 \\
\hline 40 & 77.2 & 19.5 & 9.5 & 1.89 \\
\hline 50 & 72.8 & 18.3 & 8.9 & 1.78 \\
\hline 60 & 68.7 & 17.3 & 8.4 & 1.67 \\
\hline 70 & 65.0 & 16.3 & 7.9 & 1.58 \\
\hline 80 & 61.6 & 15.4 & 7.5 & 1.49 \\
\hline 90 & 58.4 & 14.6 & 7.1 & 1.41 \\
\hline 100 & 55.5 & 13.9 & 6.7 & 1.33 \\
\hline
\end{tabular}

much more laborious ab initio molecular orbital methods, like SCF Hartree-Fock, DFT, MP2, etc. Another advantage is a lesser number of assumptions than in the case of ab initio methods. Only well known fundamental parameters like ion radii, size of water molecule and its dipole moment was used in the electrostatic model. There is no need to apply a scaling factor to the experimental data in this model. Also small computers of class PC are sufficient for calculation by this model.

However, the electrostatic model could give no answers regarding precise cluster structure of hydrated ions, because water-water interactions are not included into calculations.

The model assumed that water dipoles are uniformly distributed around the ion, due to their mutual repulsions.

In summary, we present a new simple and effective model for calculation of the reduced partition function ratios for hydrated ions and the results of $1000 \cdot \ln \beta$ calculation for $\mathrm{Li}, \mathrm{K}, \mathrm{Mg}$ and $\mathrm{Ca}$ hydrated cations in temperature range from 0 to $100{ }^{\circ} \mathrm{C}$. The same model can be also applied to other hydrated ions (spherically symmetric) like $\mathrm{Cl}^{-}$and $\mathrm{Br}^{-}$, and $\mathrm{S}^{2-}$, but not to $\mathrm{Fe}^{2+}, \mathrm{Fe}^{3+}, \mathrm{Cu}^{2+}$ etc., which do not have closed electronic shells alike in noble gas atoms.

\section{Conclusions}

Inasmuch as $\beta$ factors obtained here for $\mathrm{Mg}$ and $\mathrm{Ca}$ are in good agreement with those obtained by more sophisticated ab initio methods (Rustad et al. 2010), our electrostatic model may be considered as an alternative tool which allows to 
estimate quickly the isotope fractionation range for a number of elements which exist in ionic form (with closed electronic shells) in water solutions.

Our model of hydrated ions was for the first time applied to such broad range of temperature and cation species including the second hydration shell in $\mathrm{Li}^{+}$ and $\mathrm{K}^{+}$ions. So far the calculated 1000.ln $\beta$ for potassium is the first estimation of the upper limit for this element in the Earth. At temperature of $273 \mathrm{~K}$ these values are $99.3 \%$ for $\left[\mathrm{Li}\left(\mathrm{H}_{2} \mathrm{O}\right)_{n}\right]^{+}$, and only $2.5 \%$ of $\left[\mathrm{K}\left(\mathrm{H}_{2} \mathrm{O}\right)_{n}\right]^{+}$. These limits for divalent metals, $\mathrm{Mg}$ and $\mathrm{Ca}$, fall between these two extremes (see Table 5 and Fig. 3).

\section{References}

Boldyrev A.I., J. Simson, P. von R. Schleyer 1993: Ab initio study of the electronic structures of lithium containing diatomic molecules and ions. - J. Chem. Phys., 99., pp. 8793-8804.

Chacko T., D. Cole..J Horita 2001: Equilibrium oxygen, hydrogen and carbon isotope fractionation factors applicable to geologic systems. - Rev. in Mineralogy \& Geochemistry, 43, pp. 1-81.

Clough S.A., Y. Beers, G.P. Klein, .S. L Rothman 1973: Dipole moment of water from Stark measurements of $\mathrm{H}_{2} \mathrm{O}, \mathrm{HDO}$, and $\mathrm{D}_{2} \mathrm{O}$. - J. Chem. Phys., 59, pp. 2254-2259.

Halas, S., P, Mackiewicz 2003: Radii of di-electron systems: $\mathrm{H}, \mathrm{He}, \mathrm{Li}^{+}, \mathrm{Be}^{+}, \mathrm{B3}+, \mathrm{C}^{4+}$. a revision of the ionic radius for lithium. - Ann. Pol. Chem. Soc. 2, pp. 909-913.

Humayun, M., R.N. Clayton 1995: Precise determination of the isotopic composition of potassium: Application toterrestial rocks and lunar soils. - Geochim. Cosmochim. Acta, 59, pp. 2115-2130.

Malenkov, G.G. 1962: Geometry of structures consisting of water molecules in hydrated crystals. - J. Struct. Chem., 3, pp. 206-226.

O'Neil, J.R. 1986: Theoretical and experimental aspects of isotope fractionation. - Rev. in Mineralogy, 16, pp. 1-40.

Pauling, L. 1960: The Nature of the Chemical Bond. - Cornell Univ. Press, Ithaca, New York.

Rustad, J.R., W.H. Casey, Q-Z. Yin E.J. Bylaska, A.R. Felmy, S.A. Bogatko, V.E. Jackson, D.A. Dixon 2010: Isotopic fractionation of $\mathrm{Mg}^{2+}(\mathrm{aq}), \mathrm{Ca}^{2+}(\mathrm{aq})$, and $\mathrm{Fe}^{2+}(\mathrm{aq})$ with carbonate minerals. Geochim. Cosmochim. Acta, 74, pp. 6301-6323.

Salejda, W., M.H. Tyc, M. Just 2002: Algebraic methods for resolving of the Schrödinger equation.Polish Scientific Publishers PWN, Warsaw. (In Polish.)

Silvestrelli, P.L., M. Parrinello 1999: Water molecule dipole in gas and in the liquid phase. - Phys. Rev. Lett., 82, pp. 3308-3311.

Singer, G., P.A. Rock 1972: Thermodynamics of lithium exchange reactions. III. Electrochemical studies of exchange between isotopic metals and aqueous ions. - J. Chem. Phys., 12, pp. 55565561.

Tomascak, P.B. 2004: Development in the understanding and application of lithium isotopes in the earth and planetary systems. - Rev. in Mineralogy \& Geochemistry, 55, pp. 153-195.

Weast, R.C. Ed. 1980: CRC handbook of chemistry and physics, 61st ed. - CRC Press Inc. 1980-1981.

Urey, H.C. 1947:The thermodynamic properties of isotopic substances. - J. Chem. Soc., 1947 (London), pp. 562- 581.

Yamaji K., Y. Makita H. Watanabe, H. Sonoda, H. Kanoh, T. Hirotsu, Ooi Kenta 2001: Theoretical estimation of lithium isotopic reduced partition function ratio for lithium ions in aqueous solution. - J. Phys. Chem., A 105, pp. 602-613. 